\title{
Chrysin promotes osteogenic differentiation via ERK/MAPK activation
}

\author{
Wenfeng Zeng ${ }^{1,2^{*}}$, Yan Yan $^{3^{*}}$, Fayun Zhang ${ }^{1}$, Chunling Zhang ${ }^{1 凶}$, Wei Liang ${ }^{1 \bowtie}$ \\ ${ }^{1}$ Protein \& Peptide Pharmaceutical Laboratory, Institute of Biophysics, Chinese Academy of Sciences, Beijing 100101, China \\ ${ }^{2}$ University of the Chinese Academy of Sciences, Beijing 100049, China \\ ${ }^{3}$ Department of Endodontics, Beijing Stomatological Hospital, Capital Medical University, Beijing 100050, China \\ $\triangle$ Correspondence: weixx@sun5.ibp.ac.cn (W. Liang), zhangcl@moon.ibp.ac.cn (C. Zhang) \\ Received January 10, 2013 Accepted March 25, 2013
}

\begin{abstract}
The effect of the anti-inflammatory flavonoid chrysin on osteogenesis was determined in preosteoblast MC3T3E1 cells. Results demonstrated that chrysin could induce osteogenic differentiation in the absence of other osteogenic agents. Chrysin treatment promoted the expression of transcription factors (Runx2 and Osx) and bone formation marker genes (Col1A1, OCN, and OPN) as well as enhanced the formation of mineralized nodules. During osteogenic differentiation, chrysin preferentially activated ERK1/2, but not JNK nor the p38 MAPKs. Further experiments with inhibitors revealed the co-treatment of U0126, PD98059, or ICI182780 (a general ER antagonist) with chrysin effectively abrogated the chrysin-induced osteogenesis and ERK1/2 activation. Thus, the effect of chrysin on osteogenesis is ERK1/2-dependent and involves ER. Therefore, chrysin has the significant potential to enhance osteogenesis for osteoporosis prevention and treatment.
\end{abstract}

KEYWORDS chrysin, osteogenesis, ERK1/2, estrogen receptor

\section{INTRODUCTION}

Bone is a living tissue that continuously undergoes turnover via bone resorption and osteoclast formation by osteoclasts and osteoblasts, respectively. Unbalanced bone turnover causes disorders such as osteoporosis. Osteoporosis is characterized by thin and brittle bones, mainly in the wrists, spine, and hips, which increase the probability of incurring fractures. The condition affects both men and women across all races, but postmenopausal women and senile individuals have exhibited higher vulnerability (Becker et al., 2010). Proven therapies for

*These authors contributed equally to the work.

(C) Higher Education Press and Springer-Verlag Berlin Heidelberg 2013 the prevention and treatment of osteoporosis include bisphosphonates, calcitonin, estrogens, teriparatide, and raloxifene (Kawai et al., 2011). Most of these treatments aim to reduce the occurrence of fractures by inhibiting bone resorption. Therefore, finding an anabolic agent that promotes osteogenesis is crucial for the treatment of osteoporosis.

Osteogenic differentiation is a tightly controlled process that is mainly regulated by transcription factors, such as runt-related transcription factor-2 (Runx2) and osterix (Osx). Runx2 and Osx control the expression of bone formation marker genes, including type I collagen (Col1A1), osteocalcin (OCN), and osteopontin (OPN). Mineral nodules are subsequently formed during the final stage of osteogenic differentiation.

Mitogen-activated protein kinases (MAPKs) are a family of serine/threonine kinases, which function as the most conservative and well-elicited intracellular signal mediators of cell proliferation, survival, apoptosis, motility, metabolism, and differentiation (Chang and Karin, 2001). Previous studies showed that the activation of ERK1/2, a group of three MAPKs, is essential for embryo stem cells to escape self-renewal and commit to lineage differentiation (Kunath et al., 2007). ERK1/2 is required for osteogenic mesenchymal stem cell differentiation and skeletal development (Lai et al., 2001; Ge et al., 2007).

Chrysin (5,7-dihydroxyflavone) is a natural flavonoid that exists in various plants and foods, such as passion flowers, mushroom, chamomile, propolis, and honey. Chrysin has been reported to possess anti-inflammatory and anti-carcinogenic properties (Woo et al., 2004; Weng et al., 2005; Shin et al., 2009; Ha et al., 2010), but it has rarely been applied in osteogenesis treatment. Propolis has been reported to retard osteoclast maturation and reduce bone resorption (Pileggi et al., 2009). Moreover, a number of flavonoids have been proven to induce osteoblastic differentiation; these include daidzein, diosmetin, genestein, hesperetin, icariin, and naringenin (Jia et al., 
2003; Hsu and Kuo, 2008; Zhao et al., 2008; Trzeciakiewicz et al., 2010; Hsieh et al., 2011; Swarnkar et al., 2012). Therefore, we hypothesized that chrysin may exert a stimulatory effect on osteogenic differentiation. This study demonstrated that chrysin promotes the osteogenic differentiation and mineralization of MC3T3-E1 cells. The regulatory effect of chrysin on Runx2 and Osx expression, as well as osteogenic differentiation, was found to be ERK1/2-dependent. Our study provides substantial evidence that chrysin possesses therapeutic potential for the treatment of osteoporosis by promoting osteoblastic differentiation.

\section{RESULTS}

Chrysin induced the osteogenic differentiation of MC3T3-E1 cells

We measured the cytotoxicity of chrysin on MC3T3-E1 cells at concentrations of $0-100 \mathrm{mmo} / \mathrm{L}$ for $72 \mathrm{~h}$ and $96 \mathrm{~h}$. Treatment with less than $50 \mu \mathrm{mol} / \mathrm{L}$ chrysin exhibited no cytotoxicity (Fig. 1A). Minimal reduced cell viability was observed only when the chrysin concentration reached $100 \mu \mathrm{mol} / \mathrm{L}(96.14 \%$ and $95.27 \%$ at $72 \mathrm{~h}$ and $96 \mathrm{~h}$, respectively, as compared with the vehicle control). Thereafter, we investigated the inductive effect of chrysin on the osteogenic differentiation of MC3T3-E1 cells. After $14 \mathrm{~d}$ of osteoinduction by the osteoinduction medium or chrysin treatment, the formation of mineralized nodules became evident in the MC3T3-E1 cells. Treatment with a low concentration of chrysin $(<10 \mu \mathrm{mol} / \mathrm{L})$ moderately increased mineralization, but treatment with $25 \mu \mathrm{mol} / \mathrm{L}$ chrysin significantly enhanced MC3T3-E1 cell mineralization (Fig. 1B and 1C).

We suspected that the inductive effect of chrysin affected the main osteogenic transcription factors. The elevated gene expression levels of Runx2 and Osx were observed throughout the $96 \mathrm{~h}$ of chrysin treatment (Fig. 2A and 2B). The Runx2 gene expression was dynamically increased until $72 \mathrm{~h}$ (by 3.69-fold, as compared with the vehicle control), whereas Osx expression peaked at $24 \mathrm{~h}$ (by 26.42 -fold, as compared to the vehicle control) and then dropped within 48-96 $\mathrm{h}$ to around 10fold that of the vehicle control. Furthermore, the expression of bone formation marker genes, such as Col1A1, OCN and OPN, reached their peak levels at $48 \mathrm{~h}$ or $72 \mathrm{~h}$ (Fig. 2C-E). These data suggested that chrysin is capable of inducing osteoblastic MC3T3-E1 cell differentiation by upregulating osteogenic transcription factors and bone formation marker genes.

\section{Chrysin enhanced ERK1/2 phosphorylation}

Previous studies implied that MAPKs participated in the regulation of osteoblastic differentiation (Xiao et al., 2000). Western blot analysis was used to detect the phosphorylation status of MAPKs after chrysin treatment. The results showed that ERK1/2 was phosphorylated within 5 min after chrysin treatment. This phosphorylation was gradually reinforced until after $15 \mathrm{~min}$, followed by a decline within 30-120 min (Fig. 3A and 3B). ERK $1 / 2$ was notably the only type of MAPK activated by the chrysin treatment, and the p38 and JNK MAPKs were not activated (Fig. 3A). These data supported the hypothesis that chrysin can activate ERK in MC3T3-E1 cells.

\section{Role of ERK1/2 in chrysin-induced MC3T3-E1 osteogenesis}

Our initial results showed that ERK $1 / 2$ could be activated by chrysin treatment within $15 \mathrm{~min}$. To determine the role of ERK1/2 in chrysin-induced osteogenesis, we antagonized the effect of chrysin using MEK-specific inhibitors that functioned as the upstream kinases of ERK1/2, namely, U0126 and PD98059. Chrysin treatment for $15 \mathrm{~min}$ caused the significant phosphorylation of ERK1/2 (Fig. 4A), but both $\mathrm{U} 0126$ and PD98059 dramatically limited the inductive effect of chrysin. After $6 \mathrm{~h}$ of co-treatment with chrysin and the MEK inhibitors, the chrysin-induced upregulation of Runx2 and Osx was abrogated (Fig. 4B). U0126 and PD98059 greatly reduced the formation of mineralized nodules after $14 \mathrm{~d}$ of chrysin induction (Fig. 4C). The abovementioned data indicated that ERK1/2 is necessary for the osteogenic differentiation of MC3T3-E1 cells.

\section{ER in chrysin-induced osteogenesis and ERK1/2 activation}

Evidence has demonstrated that estrogen and estrogen receptors have important functions during osteogenesis (Kousteni et al., 2001; Khosla et al., 2011). Estrogen is considered an extracellular stimulus that activates the ERK $1 / 2$ signaling pathway. The phytoestrogenic properties of numerous flavonoids have likewise been revealed (Jungbauer and Medjakovic, 2013). Thus, we hypothesized that chrysin could be a phytoestrogen that can activate ERK1/2 via estrogen receptors. Co-treatment with the general estrogen receptor antagonist ICI182780 retarded mineralization after $14 \mathrm{~d}$ of chrysin treatment (Fig. 5A). Co-treatment of chrysin with ICl182780 for indicated periods of time $(15,60$, and $120 \mathrm{~min})$ impaired ERK1/2 phosphorylation (Fig. 5B), thereby indicating that ER is necessary for chrysininduced ERK1/2 activation. Furthermore, the gene expression levels of Runx2, Osx, Col1A1, OCN, and OPN were significantly downregulated by ICl182780 treatment (Fig. 5C and 5D). Our data further confirmed that ER is involved in chrysin-induced osteogenesis.

\section{DISCUSSION}

This study demonstrates for the first time that chrysin has potential to induce osteogenic differentiation and promote maturation in MC3T3-E1 cells. The underlying molecular mechanism shows that ERK $1 / 2$ is responsible for the inductive effects of chrysin. Previous studies demonstrated the involvement of ERK1/2 in osteoblast differentiation, but its role remained debatable (Schindeler and Little, 2006). ERK1/2 was reported to be involved in the ascorbic acid-induced $\mathrm{OCN}$ expression and the BMP-4-induced OCN secretion (Xiao et al., 2000; Kozawa et al., 2002). The ERK-dependent OCN and OPN expression 
A

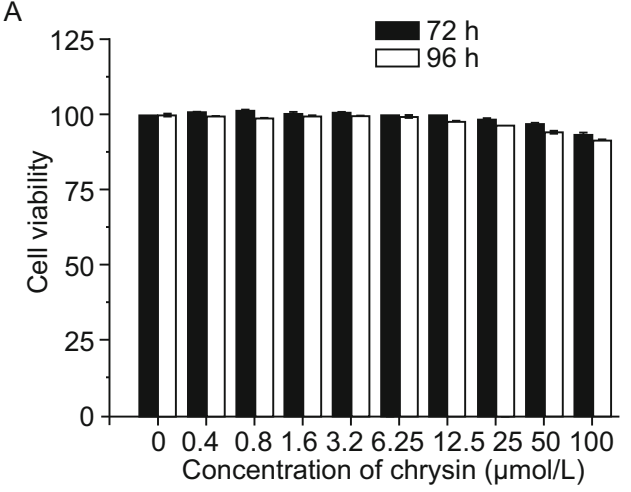

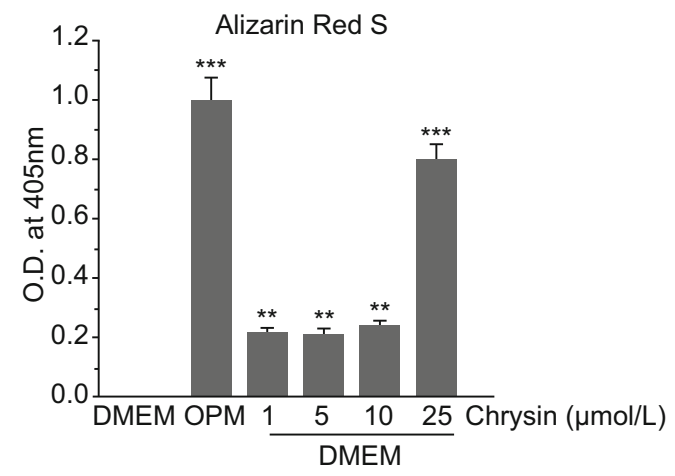

DMEM + Chrysin $(\mu \mathrm{mol} / \mathrm{L})$
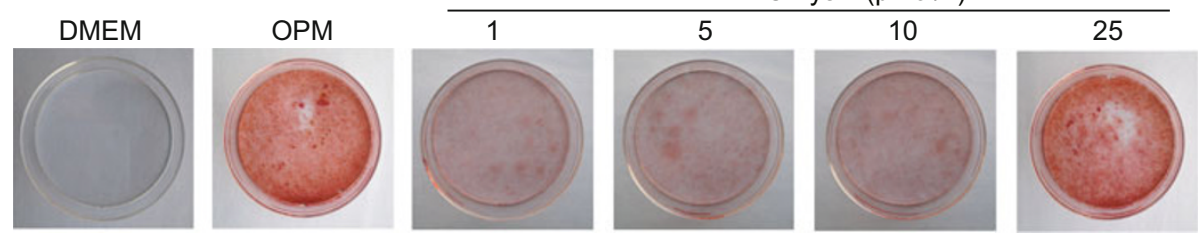

Figure 1. Effects of chrysin on the osteogenesis of MC3T3-E1 cells. Cells were treated with chrysin $(0-100 \mu \mathrm{mol} / \mathrm{L})$ for $72 \mathrm{~h}$ and $96 \mathrm{~h}$. Cell viabilities were evaluated by LDH assays (A). Cells were treated with $1,5,10$, and $25 \mathrm{mmol} / \mathrm{L}$ chrysin or osteoinduction medium (OPM) for $14 \mathrm{~d}$, and then stained with Alizarin Red S (C). Cells were lysed to release the dye, and the absorbance was measured at $405 \mathrm{~nm}$ (B). All data are presented as mean \pm SD and representative of at least three independent experiments. ${ }^{* *} P<0.01$ and ${ }^{* * *} P<0.005$ relative to the DMEM control.

implied its regulatory function in the late phase of osteoblast differentiation. By contrast, ERK inhibition by pharmacological or genetic methods improved osteogenesis and mineralization in MC3T3-E1 or C2C12 cells (Higuchi et al., 2002; Nakayama et al., 2003). The present study demonstrated that ERK1/2 activation upregulated the expression of bone formation marker genes (Col1A1, OCN, and OPN) and enhanced the mineralized nodule formation in chrysin-treated MC3T3-E1 cells.

Osteogenesis is tightly controlled by major transcription factors, including Runx2 and Osx. No bone formation was observed in Runx2 or Osx null mice, thereby suggesting that Runx2 and Osx are both required for osteoblast differentiation (Komori et al., 1997; Nakashima et al., 2002). Together with other transcription factors, Runx2 could induce cell differentiation into chondrocytes or osteoblasts, whereas Osx stimulated progenitor cells to osteoblastic commitment (Franceschi et al., 2007). In the present study, we showed that Osx induction by chrysin was more prominent than that of Runx2 within $24 \mathrm{~h}$, while chrysin significantly activated ERK1/2 (Fig. S1). Previous reports showed that Runx2 could be phosphorylated by ERK $1 / 2$ both in vitro and in vivo (Xiao et al., 2000) and Osx expression was affected by ERK1/2 activity (Celil and Campbell, 2005). Thus, the inductive effect of chrysin on Osx and Runx2 expression could be attributed to ERK1/2 activation. The inhibition of ERK by U0126 or PD98059 attenuated the inductive effect of chrysin on Osx expression and mineralization. However, the chrysin-induced Runx2 expression was reduced to a lesser extent by ERK, as compared with Osx inhibition. This difference may be explained by the post-translational regulation of Runx2 by ERK. Collectively, our findings agree with that ERK $1 / 2$ is necessary for osteogenic differentiation. Furthermore, chrysin-induced osteogenesis is ERK $1 / 2$ dependent.

During osteoblast differentiation, several extracellular factors participate in this process, including estrogen, $\mathrm{PTH}, \mathrm{IHH}$, IGF-1, and the BMPs. Bone is an estrogen-related organ, whose development needs estrogen/ER signaling regulation. Chrysin is a flavonoid, which can act as a phytoestrogen. Thus, the phytoestrogenic effect of chrysin via ER is a reasonable hypothesis. Our results showed that the ER antagonist ICl182780 substantially abrogated chrysin-induced expression of Runx2, Osx, and the bone formation marker genes (Col1A1, OCN, and OPN), as well as suppressed the formation of mineralized nodules. Furthermore, ICI182780 efficiently reduced the chrysin-induced ERK1/2 activation. All these findings suggest that chrysin acts as a phytoestrogen to induce the osteogenic differentiation of preosteoblast MC3T3-E1 cells via ERK activation.

Apart from estrogen, endogenous BMPs that accumulate in the extracellular matrices are critical for osteoblast differentiation (Suzawa et al., 1999). We measured the expression of BMP-4 under chrysin treatment in a time-dependent manner. The chrysin-induced BMP-4 expression occurred at a relatively early time point $(6 \mathrm{~h})$ and lasted until $72 \mathrm{~h}$ (Fig. S2). Previous studies indicated that BMP-4 mediated osteoblastic differentiation via crosstalk with ERK1/2 signaling (Kozawa et al., 2002). Thus, we hypothesize another plausible mechanism of chrysin 


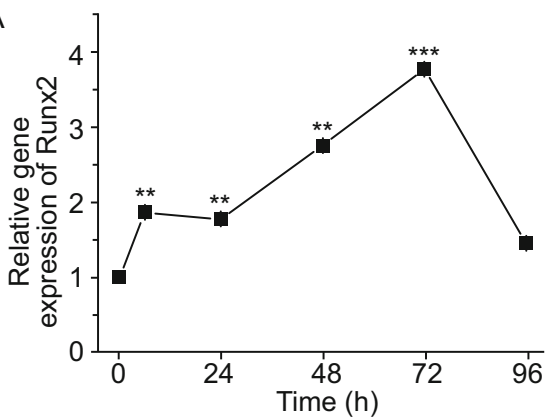

C

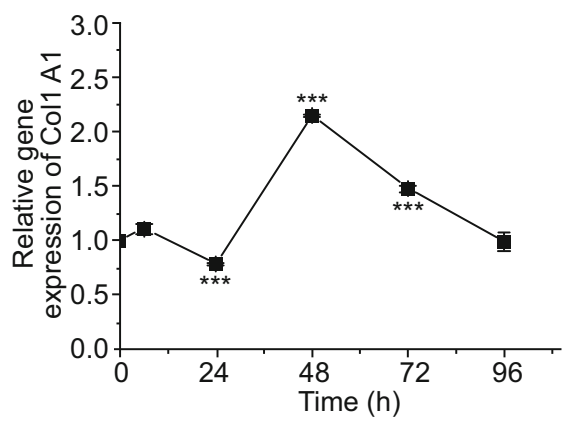

$E$

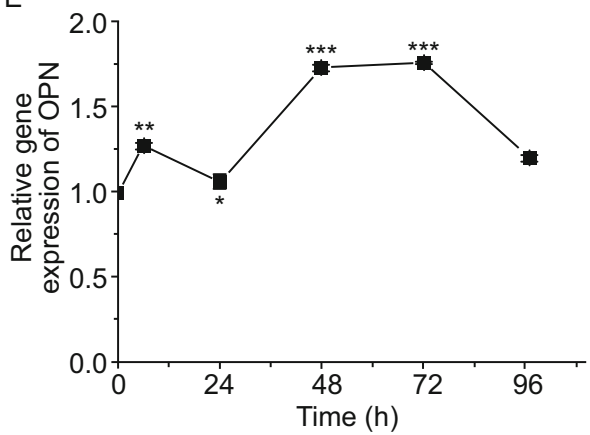

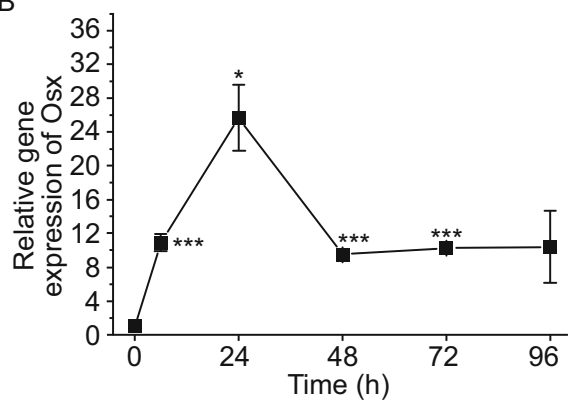

D

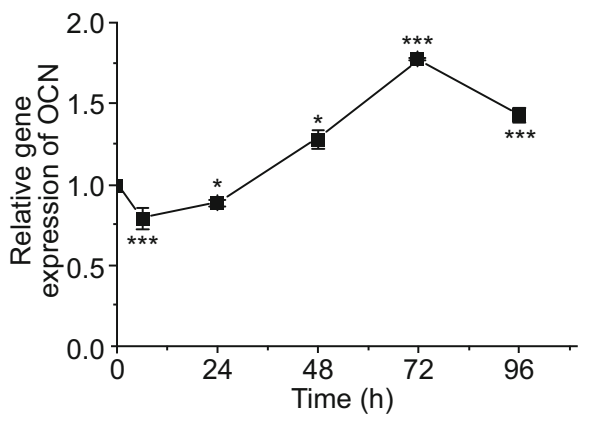

Figure 2. Effects of chrysin on the expression of osteogenesis-related genes. MC3T3-E1 cells were treated with $25 \mu \mathrm{mol} / \mathrm{L}$ chrysin for specified time periods $(6,24,48,72$, and $96 \mathrm{~h})$. Relative expression levels of the transcription factors Runx2 (A) and Osx (B) as well as the bone formation marker genes Col1A1 (C), OCN (D), and OPN (E) were detected by qRTPCR. All data are presented as mean \pm SD and representative of at least three independent experiments. ${ }^{*} P<0.05$, ${ }^{* \star} P<0.01$, and ${ }^{* *} P<0.005$ relative to the DMEM control.
A

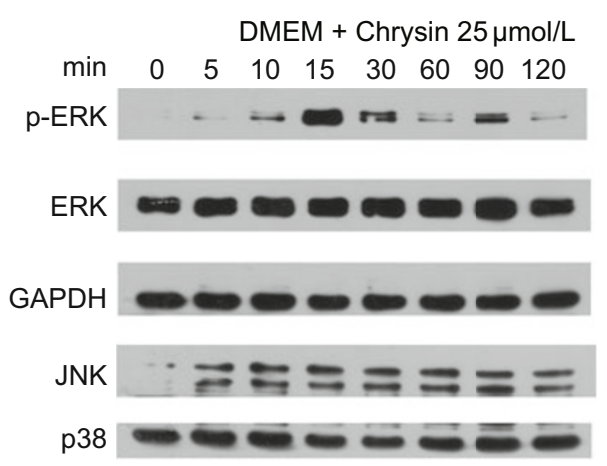

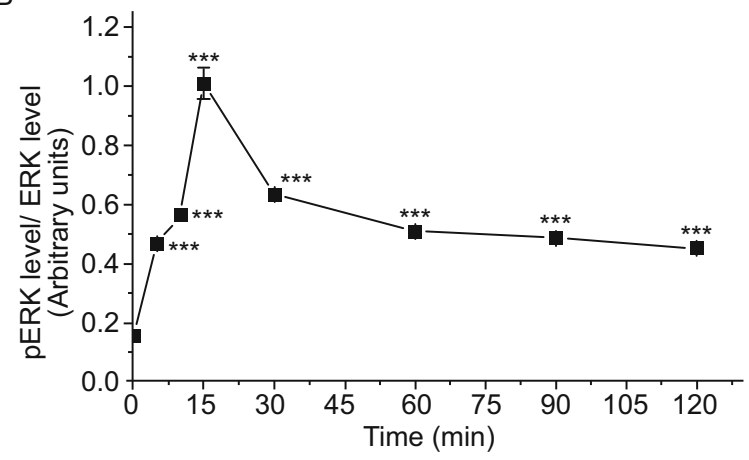

Figure 3. Chrysin induces ERK1/2 phosphorylation. MC3T3-E1 cells were treated with $25 \mathrm{mmol} / \mathrm{L}$ chrysin for specified time periods $(5,10,15,30,60,90$, and $120 \mathrm{~min})$. The phosphorylated ERK1/2, ERK1/2, JNK, P38, and GAPDH were detected by Western blot analysis (A) and the relative expression of phosphorylated ERK1/2 was quantified using the Image $\mathrm{J}$ software (B). All data are presented as mean \pm SD and representative of at least three independent experiments. ${ }^{* \star} P<0.005$ relative to the DMEM control. 
A

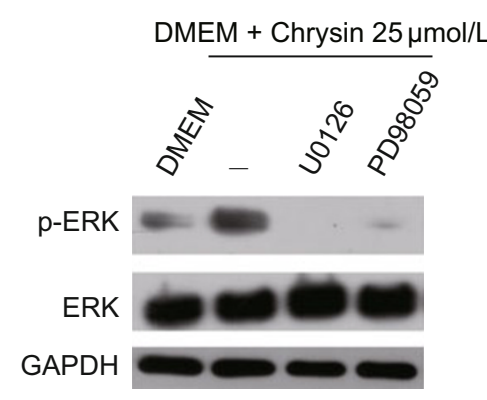

B

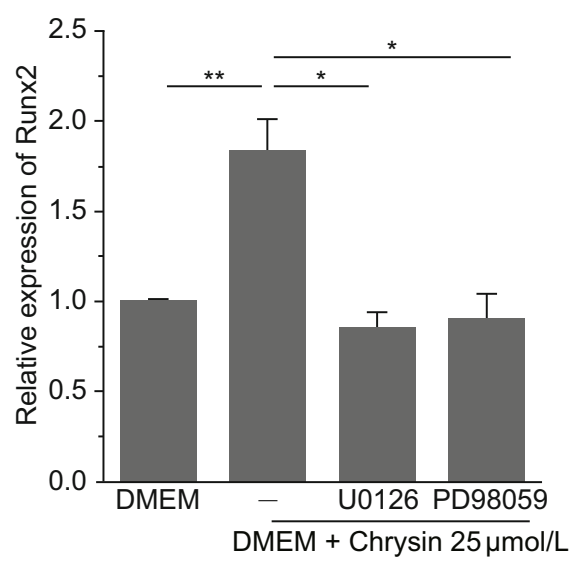

C

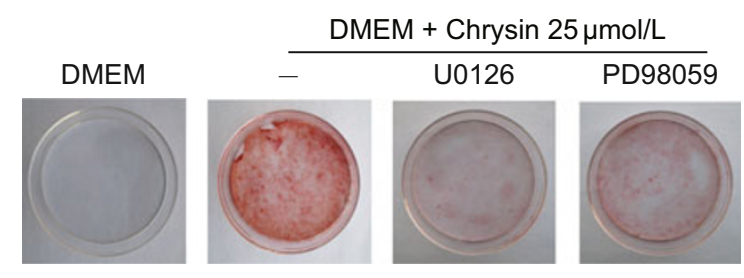

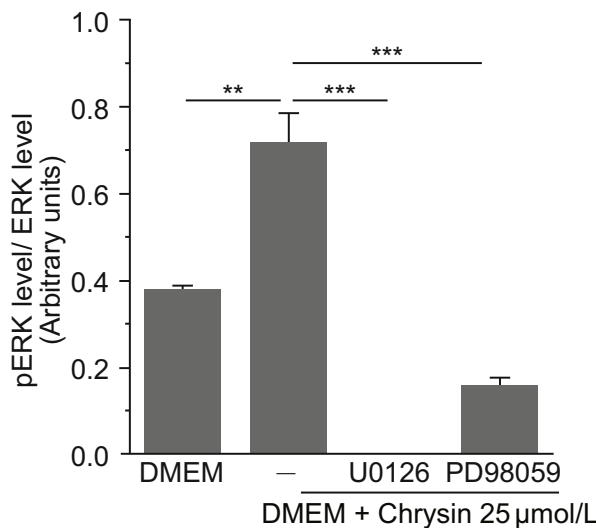
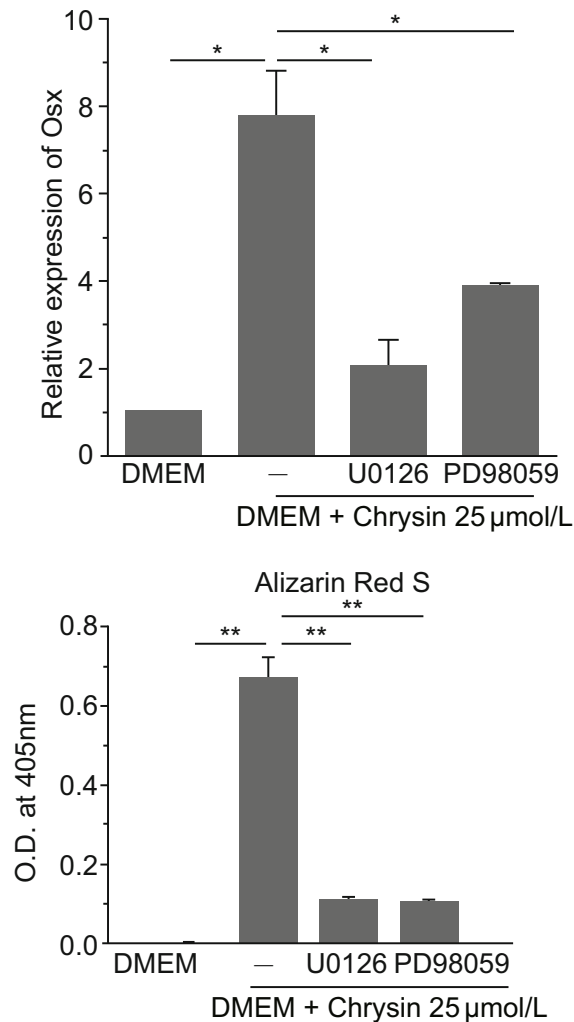

Figure 4. Role of ERK1/2 in the chrysin-induced osteogenesis of MC3T3-E1 cells. Cells were treated with $25 \mathrm{mmol} / \mathrm{L}$ chrysin with or without $10 \mu \mathrm{mol} / \mathrm{L}$ U0126 or $10 \mu \mathrm{mol} / \mathrm{L}$ PD98059 for $15 \mathrm{~min}$. The phosphorylated ERK1/2, ERK1/2 and GAPDH were detected by Western blot analysis and the relative expression of phosphorylated ERK1/2 was quantified using the Image $\mathrm{J}$ software (A). Expression of Runx2 and Osx were detected by qRT-PCR after $6 \mathrm{~h}$ of $25 \mu \mathrm{mol} / \mathrm{L}$ chrysin treatment with or without $10 \mu \mathrm{mol} / \mathrm{L}$ U0126 or $10 \mu \mathrm{mol} / \mathrm{L}$ PD98059 (B). After $14 \mathrm{~d}$ of $25 \mu \mathrm{mol} / \mathrm{L}$ chrysin treatment with or without $10 \mu \mathrm{mol} / \mathrm{L} \mathrm{U} 0126$ or $10 \mathrm{mmol} / \mathrm{L}$ PD98059, the formation of mineralized nodules was visualized by Azilian Red $S$ staining and quantified by measuring the absorbance at $405 \mathrm{~nm}$ (C). All data are presented as mean \pm SD and representative of at least three independent experiments. ${ }^{*} P<0.05$, ${ }^{* *} P<0.01$, and ${ }^{* * *} P<0.005$ relative to the DMEM plus chrysin group.

activity, that is, chrysin-induced BMP-4 expression creates an autocrine loop to boost the osteogenic differentiation of MC3T3-E1 cells.

Osteoporosis commonly occurs in post-menopausal women because of the lack of estrogen. Insufficient estrogen causes direct bone resorption, as well as indirect inflammation- induced bone loss. Current therapies for osteoporosis are mainly antiresorptive agents, except for the anabolic agent teriparatide, which is a recombinant version of human PTH (Kawai et al., 2011). However, the final objective of current osteoporosis treatment is to restore the balance between bone resorption and bone formation. Estrogen is beneficial for 

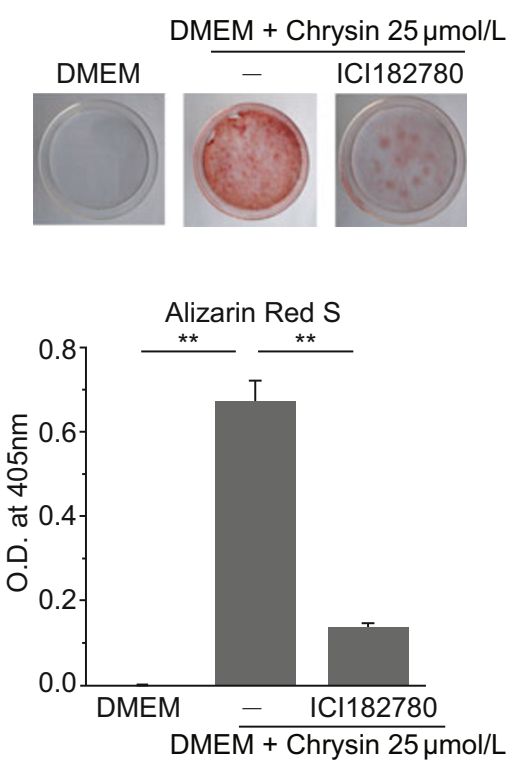

C

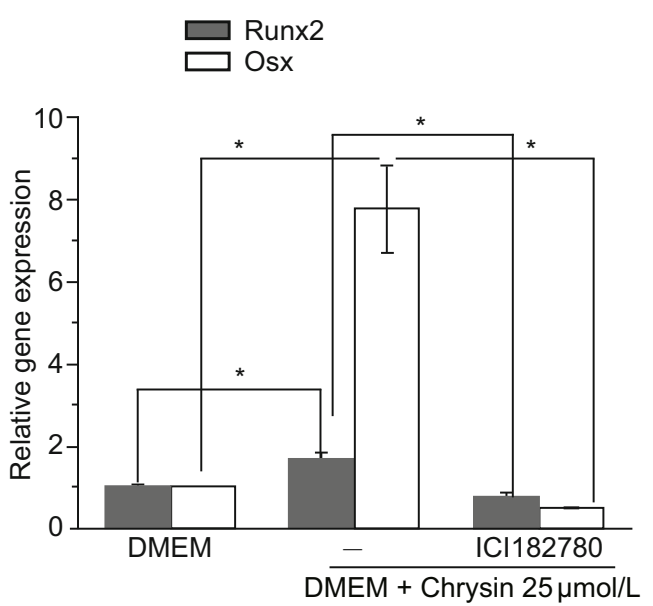

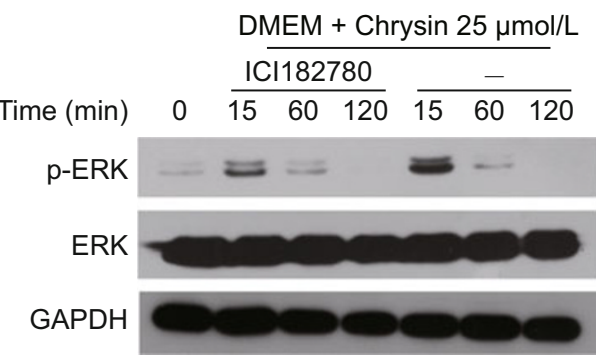
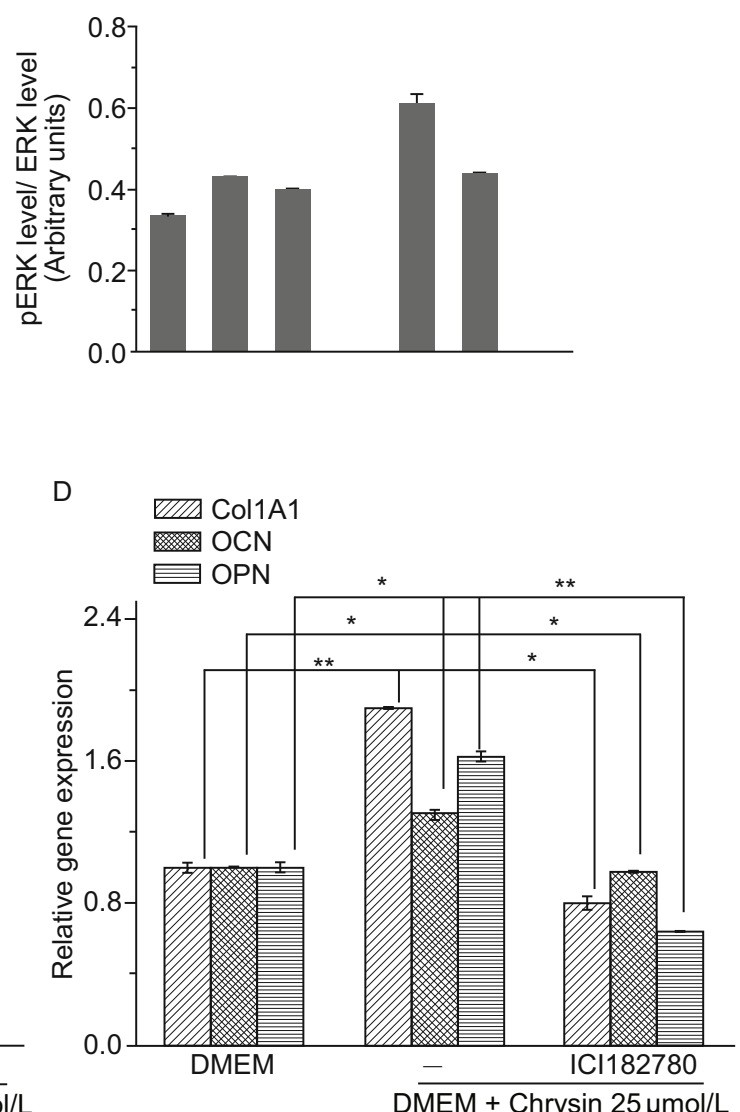

Figure 5. ER is involved in the chrysin-induced osteogenesis of MC3T3-E1. MC3T3-E1 cells were treated with $25 \mu \mathrm{mol} / \mathrm{L}$ chrysin with or without $10 \mu \mathrm{mol} / \mathrm{L} \mathrm{ICl} 182780$ for $14 \mathrm{~d}$. The formation of mineralized nodules was visualized by Alizarin Red S staining and quantified by measuring the absorbance of lysed cells at $405 \mathrm{~nm}(\mathrm{~A})$. Cells were treated with $25 \mu \mathrm{mol} / \mathrm{L}$ chrysin with or without $10 \mu \mathrm{mol} / \mathrm{L}$ ICl182780 for specified time periods (15, 60, and $120 \mathrm{~min}$ ). The phosphorylated ERK1/2, ERK1/2, and GAPDH were detected by Western blot analysis, and the relative expression of phosphorylated ERK1/2 was quantified using the Image $J$ software (B). The relative expression of the transcription factors (Runx2 and Osx) (C) and the bone formation marker genes (Col1A1, OCN, and OPN) (D) were detected by qRT-PCR under $25 \mathrm{mmol} / \mathrm{L}$ chrysin treatment with or without $10 \mu \mathrm{mol} / \mathrm{L} \mathrm{ICl} 182780 \mathrm{for} 6 \mathrm{~h}$ or $48 \mathrm{~h}$. All data are presented as mean \pm SD and representative of at least three independent experiments. ${ }^{*} P<0.05,{ }^{* *} P<0.01$, and ${ }^{* \star *} P<0.005$ relative to the DMEM plus chrysin group.

maintaining bone formation and protecting osteoblasts from apoptosis. However, estrogen treatment increases the risk of cardiovascular diseases and breast cancer, even though it could reduce the risk of osteoporotic fractures (Rossouw et al., 2002; Penckofer et al., 2003). Thus, therapeutic agents with the advantages of estrogen but with limited adverse effects are of much interest. Phytoestrogens such as flavonoids can pro- mote osteoblastic differentiation. Naringenin previously demonstrated an estrogen-like effect on osteogenesis (Swarnkar et al., 2012). Daidzein, hesperetin, and icariin were likewise reported to enhance rat osteoblast differentiation by increasing BMP production (Jia et al., 2003; Zhao et al., 2008; Trzeciakiewicz et al., 2010). Our study demonstrated that chrysin shared the reported pro-osteogenic mechanism of other flavonoids. 
However, further study on the anti-osteoclastogenic effect of chrysin is still necessary.

In conclusion, chrysin can induce the osteogenic differentiation of MC3T3-E1 cells mainly by activating ERK1/2 and ER. Chrysin may likewise have possible BMP-4-mediated autocrine effects. This finding enriches our understanding on the pro-osteogenic effect of chrysin and suggests its great potential use in the prevention or treatment of osteoporosis.

\section{MATERIALS AND METHODS}

Cell culture and treatment groups

The murine osteoblastic MC3T3-E1 cell line (kindly provided by Dr. Shujun Song from the 306th Hospital of People's Liberation Army, Beijing, China) exhibited high levels of known osteoblast markers and formed bone nodules after several days of osteoinduction in a growth medium with ascorbic acid (Xiao et al., 2002). The cells were maintained in DMEM supplemented with $10 \%$ FBS at $37^{\circ} \mathrm{C}$ in a humidified atmosphere with $5 \% \mathrm{CO}_{2}$. The culture medium was changed every $3 \mathrm{~d}$ and the cells were subcultured using a ratio of 1:6.

To prepare the cultures for osteogenic differentiation experiments, $5 \times 10^{4}$ cells were seeded in $6 \mathrm{~cm}$ culture dishes. The cells were treated for $14 \mathrm{~d}$ with either the osteoinduction medium (DMEM containing $10 \%$ FBS supplemented with $10 \mathrm{mmol} / \mathrm{L} \beta$-glycerophosphate and $50 \mu \mathrm{g} / \mathrm{mL}$ ascorbic acid) or $25 \mu \mathrm{mol} / \mathrm{L}$ chrysin. The medium was changed every $3 \mathrm{~d}$.

To prepare the cultures for RNA and protein extraction, the cells were seeded in 6-well culture plates in the required cell density. The cells were treated with $25 \mu \mathrm{mol} / \mathrm{L}$ chrysin, as well as with or without inhibitors, for specified time periods.

\section{Cell viability assay}

The cells were seeded in 96-well culture plates at a density of $35 \times 10^{3} /$ well, and treated with different concentrations of chrysin (0-100 $\mu \mathrm{mol} / \mathrm{L})$. Chrysin was dissolved in DMSO, further diluted with DMEM medium, and sterilized using a $0.22 \mathrm{~mm}$ filter. The cell viability was assessed at specified time points using LDH assays (Promega), according to the kit manufacturer's instructions.

\section{Analysis of mineralization}

After $14 \mathrm{~d}$ of osteoinduction, the cells were fixed with $4 \%$ paraformaldehyde for $30 \mathrm{~min}$ and then stained with $40 \mathrm{mmol} / \mathrm{L}$ Alizarin Red $\mathrm{S}$ for $5 \mathrm{~min}$ at room temperature. Thereafter, the cells were visualized by microscopy and their images were taken. To quantify the results, $1 \mathrm{~mL}$ lysis buffer (acetic acid:glycerol $=5: 1, v / v$ ) was added to each well, and the absorbance was measured at $405 \mathrm{~nm}\left(\mathrm{OD}_{405}\right)$.

\section{RNA extraction and qRT-PCR}

The total RNA was isolated using TRIzol reagent (Invitrogen, Life Technology), and reverse transcription PCR was performed using the Transgen TransScript one-Step gDNA Removal and cDNA Synthesis SuperMix according to the manufacturer's instructions. Quantitative real-time PCR (qRT-PCR) was performed with the Transgen TransSmart ${ }^{\mathrm{TM}}$ Green qPCR SuperMix UDG using an ABI 7300 PCR machine. The primer pairs were used as shown in Table 1.

\section{Whole cell lysate preparation and Western blot analysis}

To prepare the whole cell lysates, the cultured cells were washed with ice-cold PBS and collected by centrifugation at $450 \mathrm{~g}$. The cell pellets were lysed in cell lysis buffer (CST) that contained a cocktail of protease and phosphatase inhibitors (Sigma). Thereafter, the cell lysates were sonicated and centrifuged at $14,000 \mathrm{~g}$ at $4^{\circ} \mathrm{C}$ for $15 \mathrm{~min}$. The supernatants were collected as the whole cell lysates and then quantified using the BCA method. For each cell lysate, $20 \mu \mathrm{g}$ protein was separated using $12 \%$ SDS-PAGE for Western blot analysis. The proteins were transferred to PVDF membrane after electrophoresis. After blocking, the membranes were incubated overnight with the respective primary antibodies at $4^{\circ} \mathrm{C}$. These primary antibodies included anti-phospho-ERK, anti-phospho-JNK, anti-phospho-P38, anti-ERK, anti-JNK, anti-P38, and anti-GAPDH antibodies. The HRP-conjugated goat-anti-rabbit second antibody was used to allow detection and exposure by the ECL plus kit (Applygen Technologies Inc.). All the antibodies were purchased from CST.

\section{Statistical analysis}

Results are presented as mean \pm SD. The two-tailed Student's $t$-test was used to compare means between groups. A $P$-value less than 0.05 was considered statistically significant.

\section{ACKNOWLEDGEMENTS}

We thank Dr. Shujun Song (from the 306th Hospital of People's Liberation Army, Beijing, China) for kindly providing the MC3T3-E1 cell line. We would also like to thank Zhenwei Yang from Laboratory of Cell Biology in the Core Facilities of the Institute of Biophysics for helping with the qRT-PCR assays.

This work is supported by the following grants: the National Natural Sciences Foundation of China (Grant No. 81173633) and the National

Table 1. The primers for qRT-PCR

\begin{tabular}{lll}
\hline & Sense & Anti-sense \\
\hline Runx2 & 5'-AACGATCTGAGATTTGTGGGC-3' & 5'-CCTGCGTGGGATTTCTTGGTT-3' \\
Osx & 5'-CCCTTCTCAAGCACCAATGG-3' & 5'-AGGGTGGGTAGTCATTTGCATAG-3' \\
Col1A1 & 5'-GCTCCTCTTAGGGGCCACT-3' & 5'-CCACGTCTCACCATTGGGG-3' \\
OCN & 5'-CCGGGAGCAGTGTGAGCTTA-3' & 5'-AGGCGGTCTTCAAGCCATACT-3' \\
OPN & 5'-AGCAAGAAACTCTTCCAAGCAA-3' & 5'-GTGAGATTCGTCAGATTCATCCG-3' \\
BMP-4 & 5'-TTCCTGGTAACCGAATGCTGA-3' & 5'-CCTGAATCTCGGCGACTTTTT-3' \\
GAPDH & 5'-AGGTCGGTGTGAACGGATTTG-3' & 5'-TGTAGACCATGTAGTTGAGGTCA-3' \\
\hline
\end{tabular}


Basic Research Program (973 Program) (No. 2011CB707705).

\section{ABBREVIATIONS}

BMPs, bone morphogenetic proteins; ER, estrogen receptor; ERK, extracellular regulated protein kinases; FGFs, fibroblast growth factors; HRS, hepatocyte growth factor-regulated tyrosine kinase substrate; $\mathrm{IHH}$, Indian hedgehog; IGF-1, insulin-like growth factor 1; JNK, c-Jun amino-terminal kinase; MAPK, mitogen-activated protein kinase; OCN, osteocalcin; Osx, osterix; PI3K, phosphatidylinositol 3-kinase; PTH, parathyroid hormone; Runx2, runt-related transcription factor 2

\section{COMPLIANCE WITH ETHICS GUIDELINES}

Wenfeng Zeng, Yan Yan, Fayun Zhang, Chunling Zhang, and Wei Liang declare that they have no conflict of interest.

This article does not contain any studies with human or animal subjects performed by any of the authors.

\section{REFERENCES}

Becker, D.J., Kilgore, M.L., and Morrisey, M.A. (2010). The societal burden of osteoporosis. Curr Rheumatol Rep 12, 186-191.

Celil, A.B., and Campbell, P.G. (2005). BMP-2 and insulin-like growth factor-I mediate Osterix (Osx) expression in human mesenchymal stem cells via the MAPK and protein kinase D signaling pathways. J Biol Chem 280, 31353-31359.

Chang, L., and Karin, M. (2001). Mammalian MAP kinase signalling cascades. Nature 410, 37-40.

Franceschi, R.T., Ge, C., Xiao, G., Roca, H., and Jiang, D. (2007). Transcriptional regulation of osteoblasts. Ann N Y Acad Sci 1116, 196-207.

Ge, C., Xiao, G., Jiang, D., and Franceschi, R.T. (2007). Critical role of the extracellular signal-regulated kinase-MAPK pathway in osteoblast differentiation and skeletal development. J Cell Biol 176, 709-718.

Ha, S.K., Moon, E., and Kim, S.Y. (2010). Chrysin suppresses LPSstimulated proinflammatory responses by blocking NF-kappaB and JNK activations in microglia cells. Neurosci Lett 485, 143-147.

Higuchi, C., Myoui, A., Hashimoto, N., Kuriyama, K., Yoshioka, K., Yoshikawa, H., and Itoh, K. (2002). Continuous inhibition of MAPK signaling promotes the early osteoblastic differentiation and mineralization of the extracellular matrix. J Bone Miner Res 17, 17851794.

Hsieh, T.P., Sheu, S.Y., Sun, J.S., and Chen, M.H. (2011). Icariin inhibits osteoclast differentiation and bone resorption by suppression of MAPKs/NF-kappaB regulated HIF-1alpha and PGE(2) synthesis. Phytomedicine 18, 176-185.

Hsu, Y.L., and Kuo, P.L. (2008). Diosmetin induces human osteoblastic differentiation through the protein kinase C/p38 and extracellular signal-regulated kinase 1/2 pathway. J Bone Miner Res 23, 949-960.

Jia, T.L., Wang, H.Z., Xie, L.P., Wang, X.Y., and Zhang, R.Q. (2003). Daidzein enhances osteoblast growth that may be mediated by increased bone morphogenetic protein (BMP) production. Biochem Pharmacol 65, 709-715.

Jungbauer, A., and Medjakovic, S. (2013). Phytoestrogens and the metabolic syndrome. J Steroid Biochem Mol Biol. (In Press).

Kawai, M., Modder, U.I., Khosla, S., and Rosen, C.J. (2011). Emerging therapeutic opportunities for skeletal restoration. Nat Rev Drug Discov 10, 141-156.

Khosla, S., Melton, L.J., 3rd, and Riggs, B.L. (2011). The unitary model for estrogen deficiency and the pathogenesis of osteoporosis: is a revision needed? J Bone Miner Res 26, 441-451.

Komori, T., Yagi, H., Nomura, S., Yamaguchi, A., Sasaki, K., Deguchi, K., Shimizu, Y., Bronson, R.T., Gao, Y.H., Inada, M., et al. (1997). Targeted disruption of Cbfa1 results in a complete lack of bone formation owing to maturational arrest of osteoblasts. Cell 89, 755-764.

Kousteni, S., Bellido, T., Plotkin, L.I., O'Brien, C.A., Bodenner, D.L., Han, L., Han, K., DiGregorio, G.B., Katzenellenbogen, J.A., Katzenellenbogen, B.S., et al. (2001). Nongenotropic, sex-nonspecific signaling through the estrogen or androgen receptors: dissociation from transcriptional activity. Cell 104, 719-730.

Kozawa, O., Hatakeyama, D., and Uematsu, T. (2002). Divergent regulation by p44/p42 MAP kinase and p38 MAP kinase of bone morphogenetic protein-4-stimulated osteocalcin synthesis in osteoblasts. J Cell Biochem 84, 583-589.

Kunath, T., Saba-El-Leil, M.K., Almousailleakh, M., Wray, J., Meloche, S., and Smith, A. (2007). FGF stimulation of the Erk1/2 signalling cascade triggers transition of pluripotent embryonic stem cells from self-renewal to lineage commitment. Development 134, 28952902.

Lai, C.F., Chaudhary, L., Fausto, A., Halstead, L.R., Ory, D.S., Avioli, L.V., and Cheng, S.L. (2001). Erk is essential for growth, differentiation, integrin expression, and cell function in human osteoblastic cells. J Biol Chem 276, 14443-14450.

Nakashima, K., Zhou, X., Kunkel, G., Zhang, Z., Deng, J.M., Behringer, R.R., and de Crombrugghe, B. (2002). The novel zinc fingercontaining transcription factor osterix is required for osteoblast differentiation and bone formation. Cell 108, 17-29.

Nakayama, K., Tamura, Y., Suzawa, M., Harada, S., Fukumoto, S., Kato, M., Miyazono, K., Rodan, G.A., Takeuchi, Y., and Fujita, T. (2003). Receptor tyrosine kinases inhibit bone morphogenetic protein-Smad responsive promoter activity and differentiation of murine MC3T3-E1 osteoblast-like cells. J Bone Miner Res 18, 827-835.

Penckofer, S.M., Hackbarth, D., and Schwertz, D.W. (2003). Estrogen plus progestin therapy: the cardiovascular risks exceed the benefits. J Cardiovasc Nurs 18, 347-355.

Pileggi, R., Antony, K., Johnson, K., Zuo, J., and Shannon Holliday, L. (2009). Propolis inhibits osteoclast maturation. Dent Traumatol 25, 584-588.

Rossouw, J.E., Anderson, G.L., Prentice, R.L., LaCroix, A.Z., Kooperberg, C., Stefanick, M.L., Jackson, R.D., Beresford, S.A., Howard, B.V., Johnson, K.C., et al. (2002). Risks and benefits of estrogen plus progestin in healthy postmenopausal women: principal results From the Women's Health Initiative randomized controlled trial. JAMA 288, 321-333.

Schindeler, A., and Little, D.G. (2006). Ras-MAPK signaling in osteogenic differentiation: friend or foe? J Bone Miner Res 21, 13311338.

Shin, E.K., Kwon, H.S., Kim, Y.H., Shin, H.K., and Kim, J.K. (2009). Chrysin, a natural flavone, improves murine inflammatory bowel diseases. Biochem Biophys Res Commun 381, 502-507.

Suzawa, M., Takeuchi, Y., Fukumoto, S., Kato, S., Ueno, N., Miyazono, K., Matsumoto, T., and Fujita, T. (1999). Extracellular matrix-asso- 
ciated bone morphogenetic proteins are essential for differentiation of murine osteoblastic cells in vitro. Endocrinology 140, 2125-2133.

Swarnkar, G., Sharan, K., Siddiqui, J.A., Mishra, J.S., Khan, K., Khan, M.P., Gupta, V., Rawat, P., Maurya, R., Dwivedi, A.K., et al. (2012). A naturally occurring naringenin derivative exerts potent bone anabolic effects by mimicking oestrogen action on osteoblasts. $\mathrm{Br} \mathrm{J}$ Pharmacol 165, 1526-1542.

Trzeciakiewicz, A., Habauzit, V., Mercier, S., Lebecque, P., Davicco, M.J., Coxam, V., Demigne, C., and Horcajada, M.N. (2010). Hesperetin stimulates differentiation of primary rat osteoblasts involving the BMP signalling pathway. J Nutr Biochem 21, 424-431.

Weng, M.S., Ho, Y.S., and Lin, J.K. (2005). Chrysin induces G1 phase cell cycle arrest in C6 glioma cells through inducing p21Waf1/Cip1 expression: involvement of p38 mitogen-activated protein kinase. Biochem Pharmacol 69, 1815-1827.

Woo, K.J., Jeong, Y.J., Park, J.W., and Kwon, T.K. (2004). Chrysin- induced apoptosis is mediated through caspase activation and Akt inactivation in U937 leukemia cells. Biochem Biophys Res Commun 325, 1215-1222.

Xiao, G., Gopalakrishnan, R., Jiang, D., Reith, E., Benson, M.D., and Franceschi, R.T. (2002). Bone morphogenetic proteins, extracellular matrix, and mitogen-activated protein kinase signaling pathways are required for osteoblast-specific gene expression and differentiation in MC3T3-E1 cells. J Bone Miner Res 17, 101-110.

Xiao, G., Jiang, D., Thomas, P., Benson, M.D., Guan, K., Karsenty, G., and Franceschi, R.T. (2000). MAPK pathways activate and phosphorylate the osteoblast-specific transcription factor, Cbfa1. J Biol Chem 275, 4453-4459.

Zhao, J., Ohba, S., Shinkai, M., Chung, U.I., and Nagamune, T. (2008). Icariin induces osteogenic differentiation in vitro in a BMP- and Runx2-dependent manner. Biochem Biophys Res Commun 369, 444-448. 